\title{
A New Application in Dermatological Ultrasound
}

\author{
Kazumi Fujioka $^{1 *}$, Akira Fujioka ${ }^{2}$, Minoru Oishi $^{3}$ and Masahiro Okada ${ }^{1}$ \\ ${ }^{1}$ Department of Radiology, Nihon University School of Medicine, Japan \\ ${ }^{2}$ Department of Dermatology, Fujioka Dermatological Clinic, Japan \\ ${ }^{3}$ Department of Internal Medicine, Izutobu General Hospital, Japan \\ *Corresponding author: Kazumi Fujioka, Department of Radiology, Nihon University School of Medicine, Japan
}

\begin{tabular}{|c|c|}
\hline ARTICLE INFO & CITATION \\
\hline $\begin{array}{l}\text { Received: 慧 November 06, } 2019 \\
\text { Published: 慧 November 13, } 2019\end{array}$ & $\begin{array}{l}\text { Citation: Kazumi Fujioka, Akira Fujioka, Minoru Oishi, Masahiro Okada. A New } \\
\text { Application in Dermatological Ultrasound. Biomed J Sci \& Tech Res 22(5)-2019. BJSTR. } \\
\text { MS.ID.003809. }\end{array}$ \\
\hline
\end{tabular}

\section{Introduction}

As the high-resolution multifrequency transducers and multichannel color Doppler machines have been developed, dermatologic ultrasound imaging has been rapidly growing in recently years [1]. Joint Ultrasonography (US) in psoriasis [2] and hair morphology by US in androgenetic alopecia [3] have been also studied in current dermatologic ultrasound. Now, acoustic coupler, namely SF001 for superficial or proximal observation has been developed [4]. The usefulness of the acoustic coupler, SF-001 has been reported in the joint US of rheumatoid arthritis [4]. We have experienced the use of acoustic coupler for the dermatologic lesion for panniculitis due to the venous insufficiency condition in the lower extremity and superficial lipoma in the thigh location. We have described the advantages of this procedure in detail.

\section{Dermatologic Ultrasound and Acoustic Coupler}

The international working group, namely DERMUS (Dermatologic Ultrasound) was formed and provided the guidelines [1] and proposed for an assessment training program [5]. The minimum frequency recommended for performing dermatologic examinations by DERMUS was $15 \mathrm{MHz}$ [5]. Peripheral joint/entheseal US in patient with psoriasis [2] and hair morphology by US in patient with androgenetic alopecia [3] have been done in current dermatologic ultrasound. We usually perform US studies for dermatologic fields with a high-resolution, broad-band (5MHz-18MHz) linear transducer (Nobulus Hitachi, Ltd.Tokyo, Japan). We have also provided several reports of the dermatologic lesion [6-15]. While, Carra et al. [16] recommended a high-frequency (12-17 MHz) linear transducer for superficial masses because of its high spatial resolution. They suggest that only light pressure should be used to avoid compressing small vessels and missing flow. They prefer use of a copious amount of gel rather than a standoff pad. They noted that the pad can be cumbersome and limit some dynamic maneuvers. Now, acoustic coupler, namely SF-001 for superficial or proximal observation has been developed [4]. The usefulness of the acoustic coupler, SF-001 has been reported in the joint US of rheumatoid arthritis [4]. We describe the experience using the acoustic coupler, namely, SF-001 for the dermatologic lesion for panniculitis due to the venous insufficiency condition in the lower extremity and superficial lipoma in the thigh location. We performed US study for the lesion with a high-resolution, broad-band (5MHz-18MHz) linear transducer equipped with acoustic coupler SF-001. In result, the author will also recommend the observation for dermatologic superficial lesion, especially, rough, unevenness part in hand, foot, and joint location. Because the characteristic of acoustic coupler has been recognized as stableness state, equipped condition, and satisfactory observation for vasculature status due to the low attenuation material.

\section{Anatomy in Dermatologic Ultrasound}

The US features of healthy skin is a layer of variable thickness depending on the site, the age and constitutional habitus of the patient. It has been suggested that the distinction between the layers of the skin is important because different skin diseases occur in different layers [17]. According to the Mandava's report [18], normal skin is consist of three layers: The epidermis (thickness $0.06-0.6 \mathrm{~mm}$ ) on the most external surface; the underlying dermis (thickness 1-4 mm) containing of connective tissue, nerves, blood, and lymphatic vessels, gland, mast cells, fibroblasts, histiocytes, etc; 
and the subcutaneous tissue (thickness $5-20 \mathrm{~mm}$ ), mainly made of adipose cells. The epidermis appears as a hyperechoic line in nonglabrous skin, while it is shown as bilaminar hyperechoic and parallel lines in glabrous skin. The dermis appears as a hyperechoic band, usually less bright than the epidermis, whereas subcutaneous fat layer appears as a hypoechoic with hyperechoic fibrous septa in between [19]. The smooth distinction between the deep dermis and the superficial dermis begins to be possible. Especially in case of photoaging skin, the superficial dermis is slightly more hypoechoic [20]. Wortsman [21] also suggested that the upper dermis in adults may show lower echogenicity, namely the subepidermal lowechogenicity band due to deposits of glycosaminoglycans produced by photoaging. More deeply, the superficial fascia covering the muscular tissues can be seen as a hyperechoic regular line [18].

\section{Panniculitis in Dermatology}

Stasis dermatitis commonly occurs in older age. It is attributed to the venous hypertension. Poorly demarcated erythematous plaques of the lower legs bilaterally, was initially present in stasis dermatitis. Stasis dermatitis is regarded as one of the spectrum of cutaneous findings that may result from chronic venous insufficient condition. It mimics cellulitis, contact dermatitis, and pigmented purpuric dermatoses [22]. Meanwhile, sclerosing panniculitis or Lipodermatosclerosis (LDS) $[23,24]$ is a chronic fibrosing status that usually occurs in middle-aged or elderly woman, often accompanying with venous insufficiency or arterial ischemia [23]. The early lesion of LDS resemble other diseases such as cellulitis, Erythema Nodosum (EN), stasis dermatitis, and mediumsized vasculitis [23-25]. Choonhakarn et al. have noted that the combination of histopathologic features that was the most reliable for the acute stage of LDS was septal and lobular panniculitis with lymphatic infiltration, extravasation of erythrocytes, and adipocyte necrosis. It is suggested that histopathological features in acute stage can mimic with EN. However, EN shows a predominantly septal panniculitis, not a mixed septal and lobular involvement as seen in LDS. While, in chronic stage, lipomembranous fat necrosis with microcyst formation, vascular stasis changes in subcutaneous tissue, and septal fibrous were predominant features. Furthermore, iron deposition or hemosiderin was also seen in subacute and chronic stages [23]. With respect to the panniculitis on US, a few English reports have been studied bibliographically [21,26,27]. It has been suggested that this entity is an inflammatory process of the fatty tissue of the hypodermis. It is classified into lobular, septal, and mixed forms according to the main location of the inflammatory compositions. Mainly lobular panniculitis is shown in lupus and neonatal fat necrosis. The most common type of mainly septal panniculitis is erythematous nodosum, mainly involving the anterior part of the legs. It has been reported that lobular panniculitis appears as a diffuse increased echogenicity of the fatty lobules on US. With respect to the septal panniculitis, it is suggested that hypoechoic thickening of the septa between the hyperechoic fatty lobules are recognized [21]. It is suggested that stasis dermatitis is accompanied with panniculitis in dairy dermatologic practice. Figure 1 shows panniculitis comprehensively diagnosed in the lower extremity location in a 62-year-old woman. The septal, showing thickening of the hypodermis with fatty lobules and thick hypoechoic septa in between the fatty tissues were shown on gray scale US (Figure 1a). There are a few blood flows signals on Fine Flow (Figure 1b). Figures 2a- 2c demonstrates US features including gray-scale US, color Doppler US, and Fine Flow in the same case using equipped with the acoustic coupler (SF-001). Particularly, the depiction of the superficial location in Figure 2 images can be seen more clearly than US features in Figure 1.

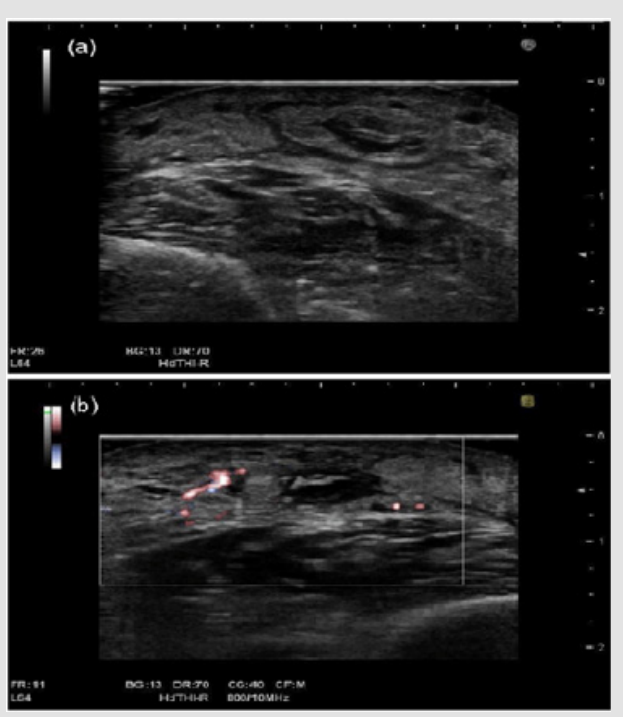

Figure 1: Panniculitis comprehensively diagnosed in the lower extremity location in a 62-year-old woman.

(a) The septal, showing thickening of the hypodermis with fatty lobules and thick hypoechoic septa in between the fatty tissues were shown on gray-scale US.

(b) There are a few blood flow signals on Fine Flow. 


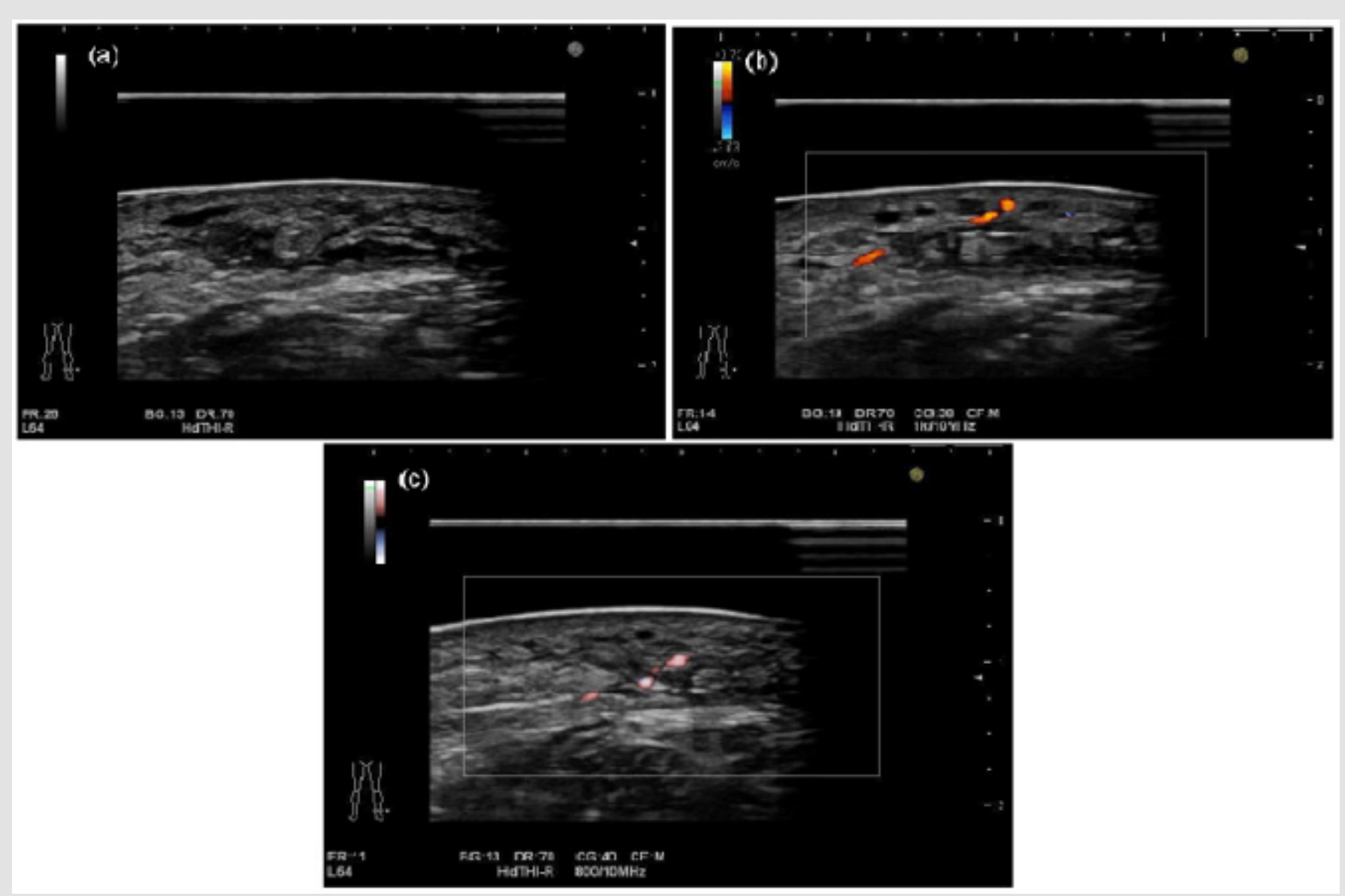

Figure 2: Panniculitis comprehensively diagnosed in the lower extremity location in a 62-year-old woman. Figure 2 demonstrated US features using equipped with acoustic coupler (SF-001).

(a) The septal, showing thickening of the hypodermis with fatty lobules and thick hypoechoic septa in between the fatty tissues were shown on gray-scale US.

(b) There are a few blood flows signals on color Doppler US.

(c) The blood flow signals on Fine Flow were also shown.

\section{Superficial Lipoma}

The author has previously reported the article of the entitled "A comparison between superficial and deep-seated lipomas on high-resolution ultrasonography: with RTE and MRI appearances" [14]. The author has also mentioned the conclusions as follows: The superficial lipoma is usually hyperechoic solid mass without posterior echo enhancement on the gray-scale ultrasonography. It is plausible that both superficial and deep-seated lipomas show echogenic lines in the mass on gray-scale ultrasonography. The superficial lipoma shows soft nature, having high elasticity and deepseated lipoma shows moderately soft nature, having moderately high elasticity on Real-Time Tissue Elastograhy. Ultrasound elastography is a technique used to depict an image of the strain on a tissue imposed by a force. Elastography is being considered as an adjunctive mode of noninvasive image in the estimation of prostate, breast, thyroid, liver tumors, and lymph nodes [28]. Figure 3 show superficial type of lipoma comprehensively diagnosed in the thigh location in a 38-year-old woman. The oblong shape with echogenic lines in the mass was detected on gray-scale US (Figure 3a). Substantial blood flow signals were not seen on color Doppler US (Figure 3b). Green color with a red color, suggesting soft nature, high elasticity on Real-Time Tissue Elastography. This figure was depicted more clearly, especially in superficial location with the acoustic coupler (SF-001) due to the stableness state, equipped condition, and low attenuation material status. 

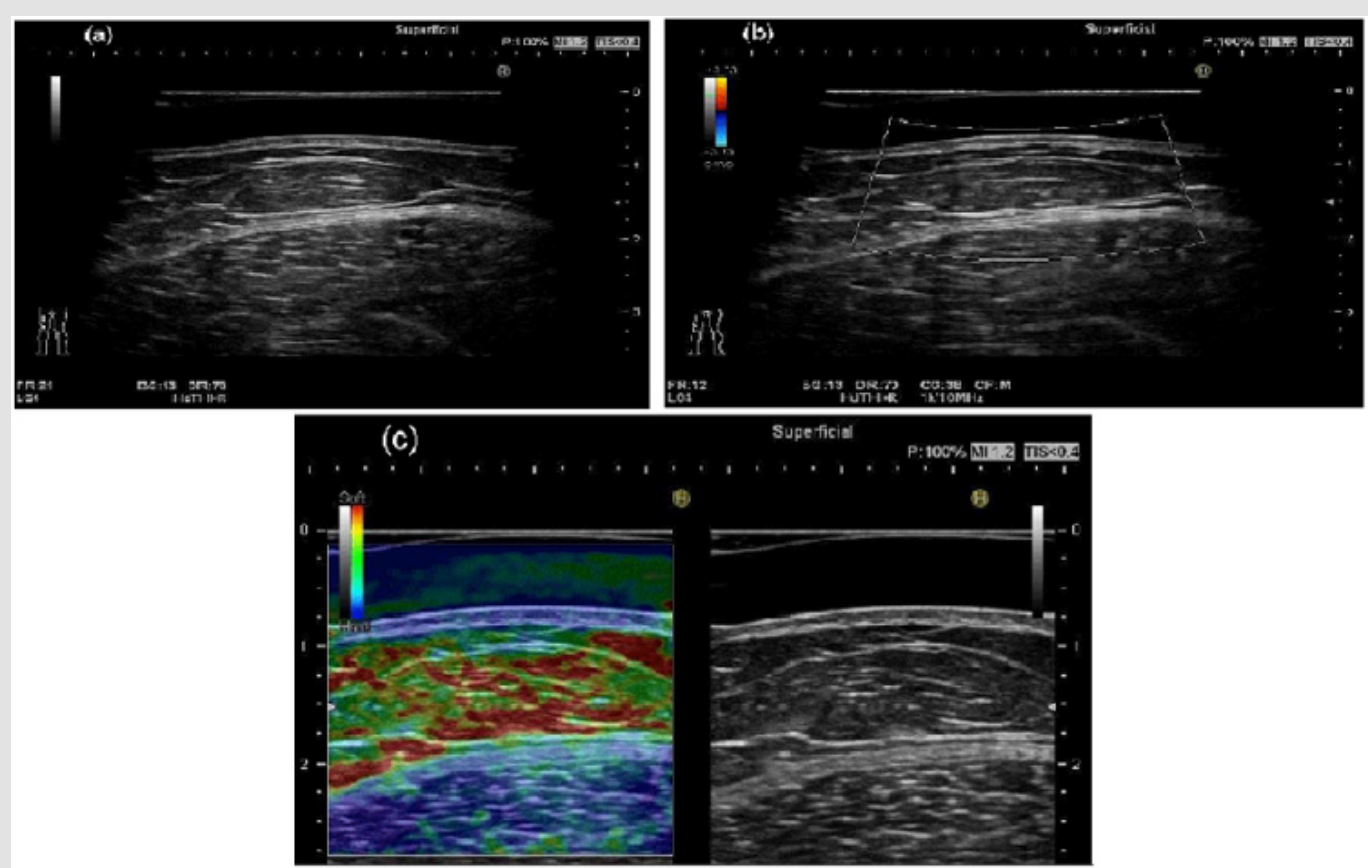

Figure 3: Superficial type of lipoma comprehensively diagnosed in the thigh location in a 38-year-old woman. Figure 3 demonstrated US features using equipped with acoustic coupler (SF-001).

(a) Gray-scale US shows the oblong shape with echogenic lines in the mass.

(b) There are no substantial blood flow signals on color Doppler US.

(c) Superficial lipoma shows green color with a red color, suggesting soft nature, high elasticity on Real-Time Tissue Elastography.

\section{Perspective in Dermatologic Ultrasound}

Soft-tissue masses in dermatology have the occurrence forms with characteristic lesion-located features. Palmar fibromatosis peripheral nerve sheath tumor, and Giant Cell Tumor of the Tendon Sheath (GCTTS) tend to occur in wrist or hand, while plantar fibromatosis, peripheral nerve sheath tumor and Morton neuroma have tendency of occurrence in ankle or foot [16]. In addition to these diseases, Fibroma of the Tendon Sheath (FTS) mostly occurs in fingers, hand, and wrists location[29]. The author will also recommend the observation with acoustic coupler for dermatologic superficial lesion, especially, rough, unevenness part in hand, foot, and joint locations. Because the usefulness of the acoustic coupler has been shown as stableness state, equipped condition, and satisfactory observation for vasculature status due to the low attenuation material.

\section{Conclusion}

1. We have described the experience using the acoustic coupler for panniculitis due to venous insufficiency and superficial lipoma.

2. The features of US image with the acoustic coupler condition is more clearly depicted, particularly in superficial location.

3. The author will also recommend the observation with acoustic coupler for dermatologic superficial lesion, especially, rough, unevenness part in hand, foot, and joint location without copious amount of gel.

4. Evaluation of the superficial location, especially for hand, foot, and joint with the acoustic coupler will be useful procedure due to the stableness, equipped, and low attenuation material conditions.

\section{References}

1. Wortsman X, Alfageme F, Roustan G, Arias-Santiago S, Martorell A, et al. (2016) Guidelines for performing dermatologic ultrasound examinations by the DERMUS group. J Ultrasound Med 35(3): 577-580.

2. Solmaz D, Bakirci S, Al Onazi A, Al Osaimi N, Fahim S, et al. (2019) Musculoskeletal ultrasound can improve referrals from dermatology to rheumatology for patients with psoriasis. Br J Dermatol https://doi. org/10.1111/bjd.18515.

3. Wortsman X, Guerrero R, Wortsman J (2014) Hair morphology in androgenetic alopecia. J Ultrasound Med 33(7): 1265-1272.

4. Sakamoto F, Narita A, Henmi M, Asano A, Tanimura K (2017) Evaluation of new acoustic coupler: SF-001 in the rheumatic ultrasound. MEDIX 67: 12-16. (Article in Japanese).

5. Wortsman X, Alfageme F, Roustan G, Arias-Santiago S, Martorell A, et al. (2016) Proposal for an assessment training program in dermatologic ultrasound by the DERMUS group. J Ultrasound Med 35: 2305-2309.

6. Fujioka K, Fujioka A, Eto H, Suzuki K, Sanuki E, et al. (2006) Nodular fascitis in the thigh followed up using ultrasonography. J Med Ultrason 33(1): 49-53.

7. Fujioka K, Fujioka A, Oishi M, Eto H, TajimaS, etal. (2017) Ultrasonography findings of intradermal nodular fasciitis; a rare case report and review of the literature. Clin Exp Dermatol 42(3): 335-336. 
8. Fujioka K, Fujioka A, Tajima S, Oishi M, Hayashi K, et al. (2018) Characteristic power Doppler sonographic imaging of nodular fasciitis from a dermatological perspective: Another case and review of three cases. J Clin Case Rep 8: 1165.

9. Fujioka K, Fujioka A, Oishi M, Hayashi K, Nakayama T. (2018) Highresolution ultrasound imaging for angioleiomyoma: A painful and vascularized superficial tumor. Biomed J Sci \& Tec Res 9(5).

10. Fujioka K (2018) Presentations of clinical, ultrasonographic and pathological features of nodular fasciitis from an established cytogenetic viewpoint: Review of the case series. J Carcinog Mutagen 9: 326.

11. Fujioka K (2018) Usefulness of comprehensive high-resolution ultrasound imaging in dermatologic field: epidermal cyst. Biomed J Sci \& Tec Res 12(1).

12. Fujioka K (2019) Painful cutaneous and subcutaneous tumors accompanied with vascularized appearance using high-resolution ultrasound in dermatology: the acronym "ENGLAND" or "LEND AN EGG". Biomed J Sci \& Tec Res 12(4).

13. Fujioka K (2019) Real- time Tissue elastography in epidermal cyst: along with enhanced MRI and elasticity features of peripheral rim. Biomed J Sci \& Tec Res 16(5).

14. Fujioka K (2019) A comparison between superficial and deep-seated lipomas on high-resolution ultrasonography: with RTE and MRI appearances. Biomed J Sci \& Tec Res 19(2).

15. Fujioka K (2019) Characteristic appearances of nodular fasciitis on high-resolution ultrasonography: with vasculature status from a lesionseated perspective. Biomed J Sci \& Tec Res 20(4).

16. Carra BJ, Bui-Mansfield LT, O’Brien SD, Chen DC (2014) Sonographic of musculoskeletal soft-tissue masses: techniques, pearls, and pitfalls. AJR AM J Roentgenol 202(6): 1281-1290.

17. Crisan D, Lupsor M, Boca A, Crisan M Badea R (2012) Ultrasonographic assessment of skin structure according to age. Indian J Dermatol Venereol Leprol 78(4): 519.

18. Mandava A, Ravuri PR, Konathan R (2013) High-resolution ultrasound imaging of cutaneous lesions. Indian J Radiol Imaging 23(3): 269-277.

\section{ISSN: 2574-1241}

DOI: 10.26717/BJSTR.2019.22.003809

Kazumi Fujioka. Biomed J Sci \& Tech Res

This work is licensed under Creative Commons Attribution 4.0 License

Submission Link: https://biomedres.us/submit-manuscript.php
19. Wortsman X (2012) Common applications of dermatologic sonography. J Ultrasound Med 31(1): 97-111.

20. Scotto di Santolo M, Saqnelli M, Mancini M, Scalvenzi M, Delfino M, et al. (2015) High-resolution color-Doppler ultrasound for the study of skin growths. Arch Dermatol Res 307(7): 559-566.

21. Wortsman X (2017) Sonography of dermatologic emergencies. J Ultrasound Med 36(9): 1905-1914.

22. Sundaresan S, Migden MR, Silapunt S (2017) Stasis dermatitis: Pathophysiology, evaluation, and management. Am J Clin Dermatol 18(3): 383-390.

23. Choonhakarn C, Chaowattanapanit S, Julanon N (2016) Lipodermatosclerosis: A clinicopathologic correlation. Int J Dermatol 55(3): 303-308.

24. Walsh SN, Santa Cruz DL (2010) Lipodermatoscerosis: A clnicopathological study of 25 cases. J Am Acad Dermatol 62(6): 10051012.

25. Bruce AJ, Bennett DD, Lohse CM, Rooke TW, Davis MD (2002) Lipodermatosclerosis: Review of cases evaluated at Mayo Clinic. J Am Acad Dermatol 46(2): 187-192

26. Habicheyn Hiar S, Segura Palacios JM, Bernal Ruiz Al (2015) Ultrasound in the management of inflammatory dermatosis. Actas Dermosifiliogr 106: 41-48.

27. Roman J, Giavedoni P, Roe E, Vidal D, Luelmo J, et al. (2019) Inter- and intra-rater agreement of dermatologic ultrasound for the diagnosis of lobular and septal panniculitis. J Ultrasound Med https://doi. org/10.1002/jum.15080.

28. Kleinerman R, Whang TB, Bard RL, Marmur ES (2012) Ultrasound in dermatology: Principles and applications. J Am Acad Dermatol 67(3): 478-487.

29. Ma X, Xu J, Wang X, Wang C, Zhang YJ (2013) Toe deformity after pregnancy due to fibroma of tendon sheath: a case report and review of the literature. Orthop Surg 5(3): 225-228.

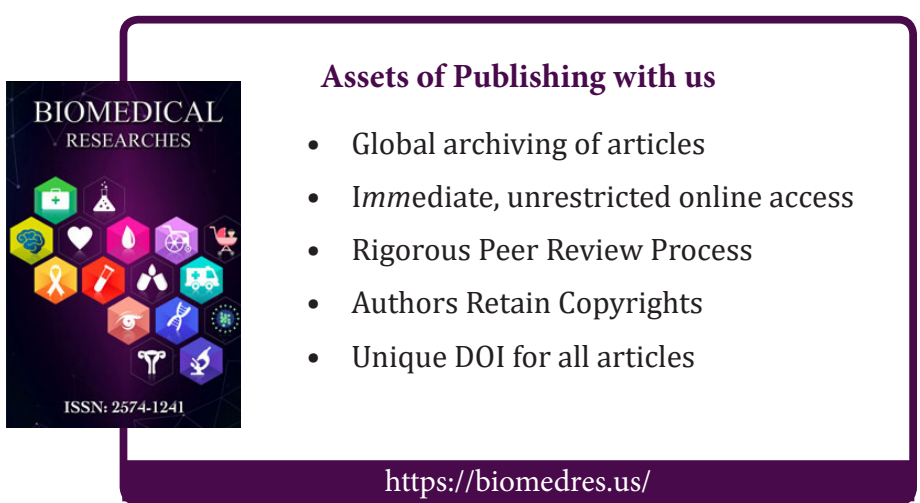

